Original Research Paper

\title{
Measurements of Mitral Annular Displacement in 2D Echocardiography Images
}

\author{
${ }^{1,2}$ Faten A. Dawood, ${ }^{2}$ Rahmita W. Rahmat, ${ }^{3}$ Suhaini B. Kadiman, ${ }^{2}$ Lili N. Abdullah and ${ }^{4}$ Mohd D. Zamrin \\ ${ }^{1}$ Department of Computer Science, College of Science, University of Baghdad, Iraq \\ ${ }^{2}$ Department of Multimedia, Faculty of Computer Science and Information Technology, UPM, 43400 Selangor, Malaysia \\ ${ }^{3}$ Department of Anaesthesiology, Institute Jantung Negara IJN hospital, Jalan Tun Razak 50400 Kuala Lumpur, Malaysia \\ ${ }^{4}$ Department of Surgery, Heart and Lung Centre HUKM, National University of Malaysia, 56000 Cheras, Malaysia
}

Article history

Received: 21-06-2014

Revised: 28-06-2014

Accepted: 15-05-2015

Corresponding Author:

Faten A. Dawood

Department of Computer

Science, College of Science,

University of Baghdad, Iraq

Email: faten_dawood12@yahoo.com

\begin{abstract}
Mitral Annular Displacement (MAD) in echocardiography has been described as a variation in mitral annulus position between the enddiastolic and the end-systolic in a complete cardiac cycle. It could be used as a rapid and reproducible method of determining the LV global systolic function and could be an easily detectable index for wall motion abnormalities. In this study, a computational method of MAD was implemented based on the mitral annulus motion tracking at both sides; namely the lateral side and the septal side using 2D-Echocardiographic (2DE) datasets. This method comprises three main processing stages: 2DE dataset preparation, Region Of Interest (ROI) selection and MAD measurements. For each $2 \mathrm{DE}$ dataset, MAD was computed as the movement distance toward the LV apex at both sides individually in twoconsecutive frames using the 'Euclidian distance' method. Then, the maximum displacement occurs during a complete cardiac cycle was measured in millimetres $(\mathrm{mm})$ for each side. The overall datasets used are 178 original 2D-echocardiography images in 4-chamber view. The experimental results for MAD measurements were compared with results that obtained by TMQ Advanced technique using QLAB software. The comparative analysis was done qualitatively by visual observation of two expert and the comparison scores show that the proposed method of MAD measurements has high acceptability of $85 \%$. Furthermore, the quantitative analysis of the MAD method is comparable with TMAD measurements by QLAB and there is no significant differences in displacement measurements.
\end{abstract}

Keywords: LV Systolic Function, Displacement Measurements, QLAB Software, Statistical Analysis, Mitral Annulus

\section{Introduction}

Echocardiography is the application of diagnostic ultrasound imaging to the heart which allow direct visualization of cardiac structure and wall motion. It has received in the evaluation of cardiac disease and in characterizing the structure and function of the heart. In clinical practice, echocardiography is widely used, noninvasive and cost-effective technique. It could be a useful diagnostic tool for assessing the abnormality of LV systolic function which based on the wall movements (Rahmat et al., 2012; Nandagopalan et al., 2010; Jagatheeswari et al., 2009). The measurement of
MAD has been considered as a rapid and reproducible method of determining the LV global systolic function and could be an easily detectable index for wall motion abnormalities (Hedberg et al., 2006). The main goal of this study is to measure the MAD based on Septal and Lateral motion tracking in a complete cardiac cycle.

A variety 2D-echocardiographic images through complete cardiac cycle was used for experimental results in many investigative researches (Dawood et al., 2011; Ahanathapillai and Soraghan, 2010; dos Reis et al., 2008; Salvador et al., 2003). Due to the anatomical structure difficulties in 2D-echocardiography images including speckle noise, low contrast and signal 
dropouts, not all images provided clear tissues of myocardial wall and in some cases, spatial filtering was needed (Sun et al., 2013; Al-Surmi et al., 2013; Dawood et al., 2012; Hoque and Al-Mahfuz, 2011; Ahmed and Nordin, 2011). In the literature, most of the common tracking methods for measuring MAD which depending on the myocardial tissue structure (Storaa et al., 2004; Pan et al., 2001). Measurement of MAD by M-mode echocardiography from apical fourchamber view has been described during recent years as a simple and reliable index for assessment the left ventricular function (Eto et al., 2005; DeCara et al., 2005; Emilsson et al., 2000). MAD was measured using tissue tracking method based on Doppler tissue imaging that allows rapid semi quantitative visual assessment of the systolic distance of tissue motion along the Doppler axis by a graded colour display (Zahid et al., 2013; Ito et al., 2007). On the other hand, two-dimensional echocardiography was performed to evaluate MAD results based on speckle tracking (Black et al., 2013; Buss et al., 2012). Tissue Mitral Annulus Displacement (TMAD) measurements are done by 'TMQ Advanced' technique using QLAB software. Three points are placed by the specialist (i.e., clinical cardiologist) in the first frame; two at septal and lateral leaflets of mitral annulus and third at the apex (Narayanan et al., 2007).

\section{Materials and Methods}

\section{DE Dataset Preparation}

In this study, 2D-echocardiography images obtained with permission from the Malaysian National Heart Institute (IJN). These images were acquired using the 'iE33 Philips' medical machine with QLAB software under the supervision of a Consultant cardiac anesthesiologist. Accordingly, the 2DE dataset acquisition and preparation stage consists of three major steps: Firstly, a matrix array transducer of TEE probe is connected to a capable echocardiography system be used on a normal subject. In the second step, all acquired data is transferred from the 'on-line' medical system directly to the 'off-line' workstation by running the QLAB software. Finally, each 2DE video broken down into its constituent frames according to the frame rate. Each of the frames are saved in the Windows BMP format. The steps in 2DE image dataset collection and preparation that were used in this study are shown in Fig. 1.

\section{ROI Selection}

Three ROI were identified manually in the first frame of 2D echocardiography video by the specialist (clinical cardiologist). The first two ROI were placed at the septal side and the lateral side of the mitral annulus and the third ROI was placed at the apex of LV. To reduce the time-consuming, the desired ROI was cropped from background and saved it in a new small image for processing. Those three ROI in apical four-chamber view are shown in Fig. 2. The selected ROI of Septal and Lateral are individually saved in a new sub-image of $40 \times 40$ pixels window size.

\section{MAD Measurements}

As mentioned earlier, the MAD measurements is a useful method that could be used to determine the global LV systolic function and a reliable index for myocardial wall motion abnormalities. Therefore, for each $2 \mathrm{DE}$ image sequences, the movement distance was computed in pixels toward the apex at both the lateral and septal sides individually in two-consecutive frames based on the Euclidian distance method as represented in Fig. 3. The parameter $P$ refers to the point in frame $f$ and the parameter $P$ refers to the point in the next frame $f+1$. The x-y coordinates for each labeled point $P$ are saved in two scalars $x P$ and $y P$ while the $\mathrm{x}-\mathrm{y}$ coordinates for each labeled point $P^{\prime}$ are saved in two scalars $x^{\square}$ and $y^{\square}$ Hence, the distance values of two points $P$ and $P$ are calculated from Equation 1 and saved in voxel $D t$ for each two consecutive frames $f$ and $f+1$ as follows:

$$
D_{t k, i}=\sqrt{\left(x P_{i}-x \square_{i}\right)+\left(y P_{i}-y \square_{i}\right)^{2}}
$$

Where:

$k=$ The number of labeled points (Lateral and Septal) $i=$ The frame number

Then, the movement distance computation will be applied to the next pair-frames and so on until the last frame. Accordingly, for each labeled point, the maximum value of displacement is measured according to the cumulative distances $D t$ in pixels for each pairframes through complete cardiac cycle. This displacement value (Dispt) is computed as in Equation 2.

$$
\text { Dispk }=\operatorname{Max}\left[\sum_{i=1}^{n} D t_{k, i}\right]
$$

Where:

$n=$ The total number of frames

$k=$ The number of labeled points (Lateral and Septal)

In order to convert the MAD measurements from pixels into millimeters (mm), it is required to know the resolution of image in dot per inch (dpi). For this purpose, a method to compute the input image resolution was proposed using the mathematical calculation based on the depth parameter which used in acquiring 2D-echocardiography images and the standard convertor formula. 


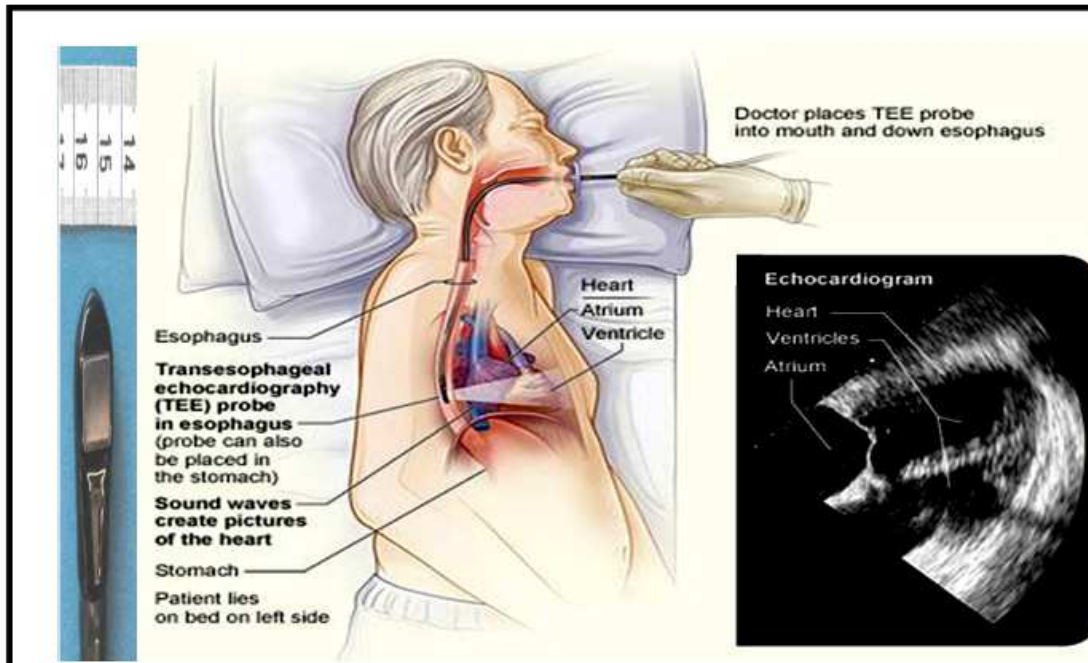

(a)

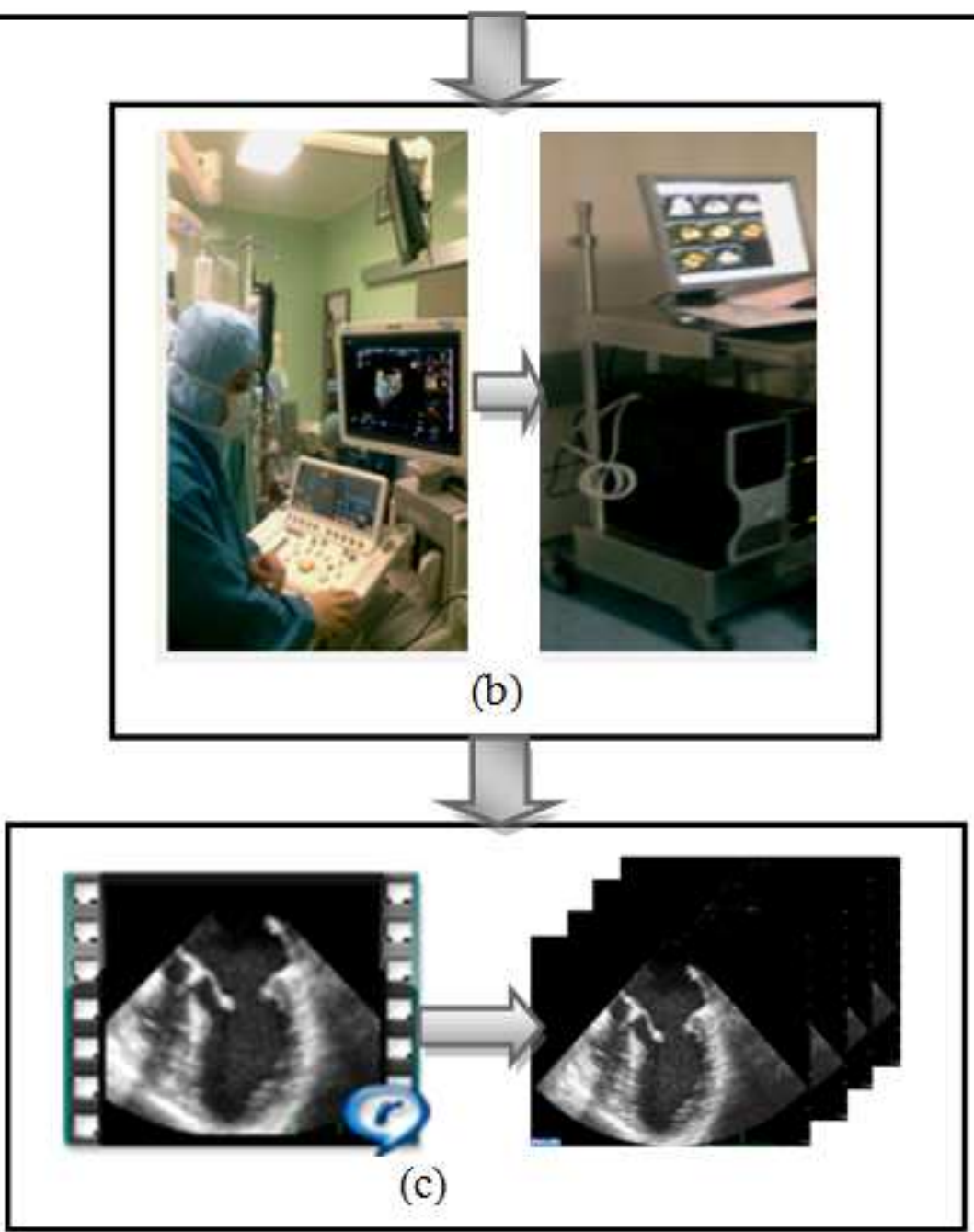

Fig. 1. Three steps of 2DE dataset collection and preparation; step1: 2DE dataset acquisition (a), step2: Dataset transformation (b) and step3: 2DE images preparation (c). Image in (a) adopted from: "National Heart, Lung and Blood Institute", 2012 


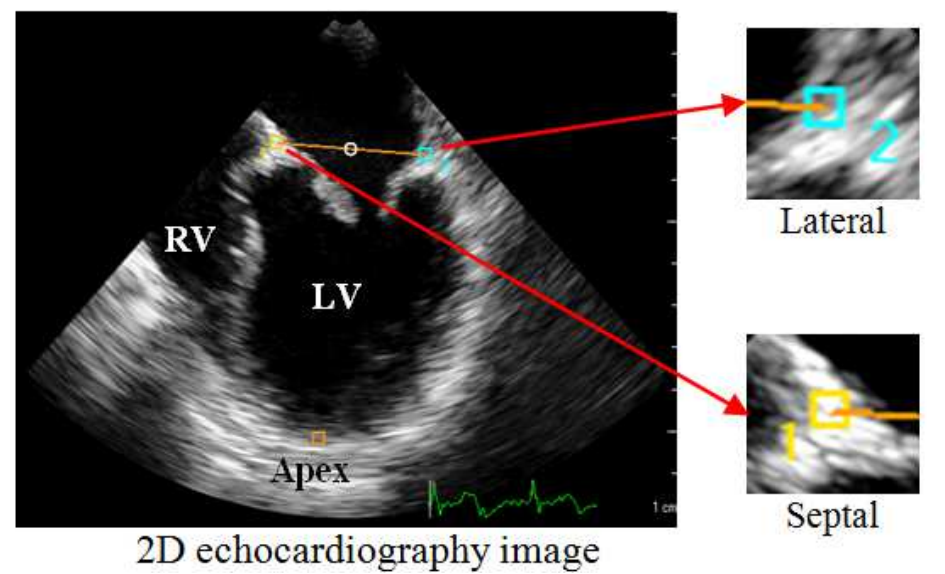

Fig. 2. Example of three ROI selection were manually placed at first frame: (1) The septal side, (2) the lateral side of the mitral annulus and (3) the apex of LV. LV, left ventricle; RV, right ventricle

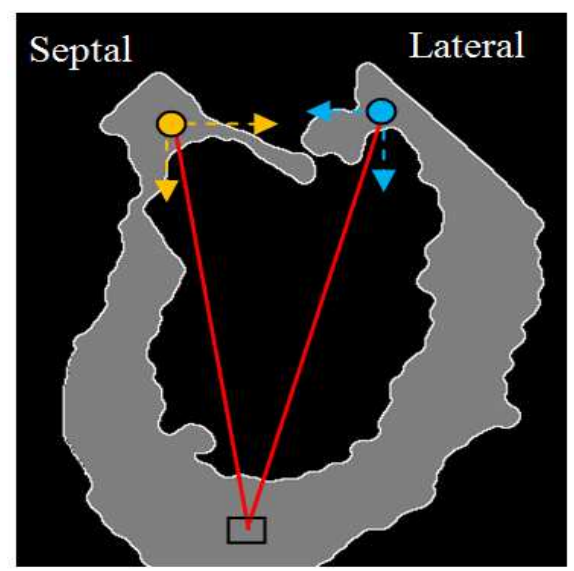

(a)

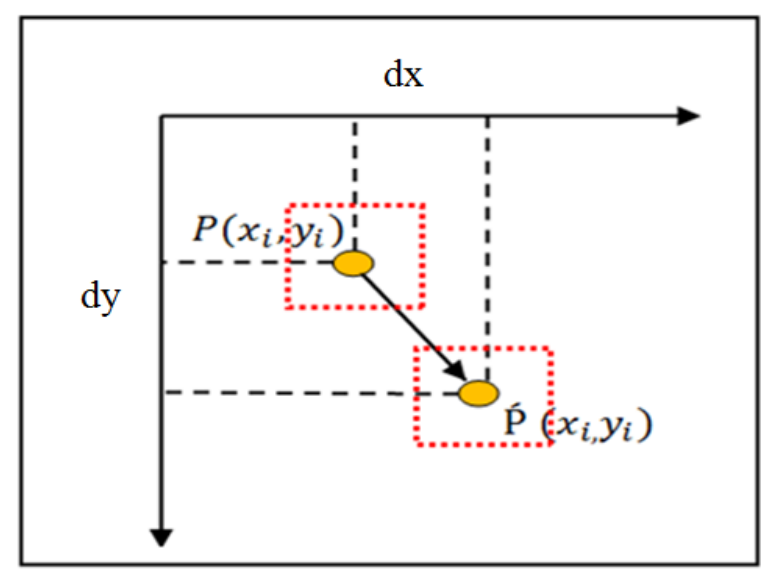

(b)

Fig. 3. The representative example of mitral annular at both sides of Septal and Lateral (a) and the computation of movement distance for each labeled-point in two-consecutive frames (b)

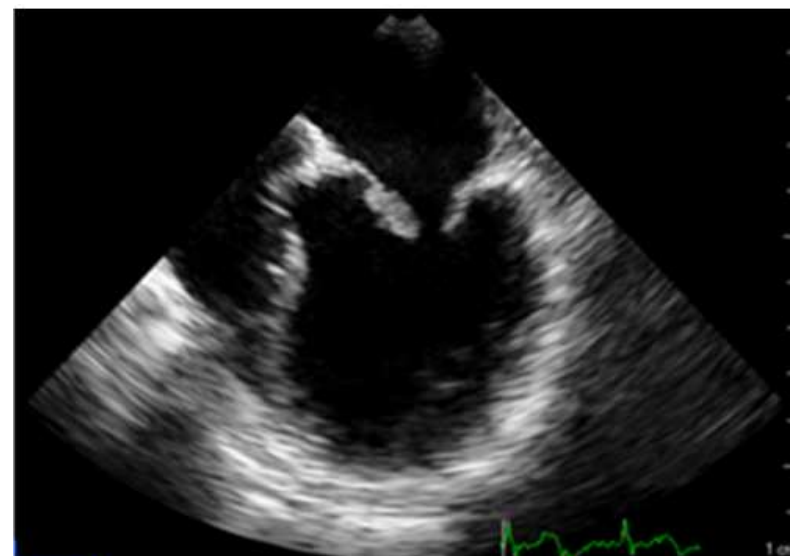

(a)

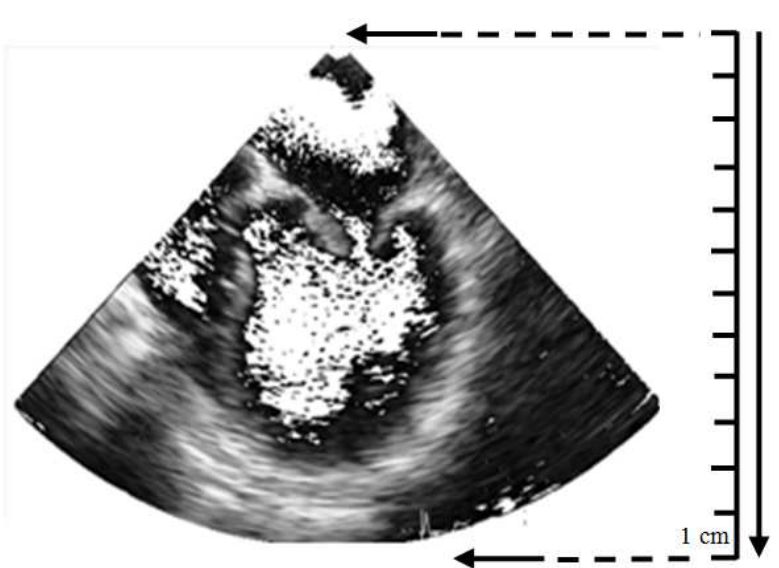

(b)

Fig. 4. Experiment of the resolution calculation based on depth parameter used in 2DE image acquisition (a) and the height $\mathrm{Y}$ of extracted ROI (b) 
In variety of acquired data, the depth parameter $(\mathrm{P})$ is different from one 2DE dataset to another such as the sample illustrated in Fig. $4 \mathrm{a}$ and has a depth of $12 \mathrm{~cm}$. Therefore, the proposed method for the resolution calculation in 2DE image comprises from three main steps:

Step1: Extract the Region Of Interest (ROI) from black background of input image, consider this ROI as the reference object and save it in a new image $\mathrm{S}$ of size $\mathrm{N} \times \mathrm{M}$ as shown in Fig. $4 \mathrm{~b}$.

Step2: Identify the height $(\mathrm{H})$ of the new image based on $\mathrm{Y}$-axis and then divide it on the depth to calculate the total pixels in each centimeter $\left(\mathrm{P}_{\mathrm{cm}}\right)$.

Step3: Compute the image resolution (dpi) according to the standard convertor formula from pixels into centimeter and thus the dpi value can be derived from Equation 3:

$d p i=\underline{(H * 2.54)}$

Where:

$\mathrm{H}=$ The height of image $\mathrm{S}$ in pixels

$\mathrm{P}=$ The depth parameter used in 2DE dataset acquisition process

Then, the displacement measurements will be converted from pixels into millimeter $(\mathrm{mm})$ by multiplying the $\mathrm{P}_{\mathrm{cm}}$ value by 10 and the new values of displacement individually saved in a profile to be used as input parameter for comparison process and statistical analysis.

\section{Results and Discussion}

As mentioned in section 2.1, all 2DE datasets were acquired using a matrix array X2-7t transducer (TEE) which connected to the medical system 'iE33 Philips' at the Malaysian National Heart Institute. To justify the accuracy of the displacement measurements based on the findings of the resolution calculation, the datasets were divided into two groups. The first group (Group1) was used for the proposed method implementation of MAD measurements which contains original 2DE videos of 4chamber views within a complete cardiac cycle for seven different patients that saved in AVI format. Each video was converted into frames (2DE images) with a total numbers of 178 2DE frames for all datasets. In the second group (Group2), the same seven 2DE videos with tissue mitral annulus displacement TMAD measurements were used to validate the performance of the proposed method via a comparative analysis. TMAD measurements have been done for all datasets using 'TMQ Advanced' technique (Narayanan et al., 2007), where QLAB has been used as a reference to validate the accuracy of the results by qualitative and quantitative assessment. Three points are marked by the specialist (clinical cardiologist) in the first frame; two at the septal and lateral leaflets of the mitral annulus and the third at the LV apex as shown in Fig. 5.

In the experiments, the Mitral Displacement Annulus (MAD) for lateral and septal points were measured using the proposed method of displacement calculations based on the $x-y$ coordinate of these two points. However, the accuracy of the experimental results depend mostly on how the proposed method successfully measures the MAD at septal side $\left(\mathrm{MAD}_{\mathrm{S}}\right)$ and lateral side $\left(\mathrm{MAD}_{\mathrm{L}}\right)$ of the LV myocardial wall. The experimental results of MAD measurements using the proposed method have been qualitavely evaluated for all datastes by two experts users; the clinical cardiologist and the cardiac technician. A comparative analysis of MAD measurements were made between the results obtained by the proposed method and the results from QLAB by 'TMQ Advanced' technique. Several examples of the experimental results for mitral annular displacement measurements with Time/curve which obtained from the proposed method and QLAB software respectively are presented in Fig. 6.

The acceptability comparison of our measurements (MAD) against QLAB measurements (TMAD) have been done according to the opinion of two experts, which is represented by a score as shown in Table 1 . The experts scores highly depended on the reliability of the representative results by the proposed method. The MAD measurements were implemented for all datasets including Groupl using the proposed method and Group2 by TMQ measurements from QLAB software.

The measurements values of $\mathrm{MAD}_{\mathrm{L}}$ and $\mathrm{MAD}_{\mathrm{S}}$ in both methods of Group1 and Group2 are listed in Table 2. For quantitative assessment, the statistical calculations by mean values \pm standard deviation (Mean \pm SD) have been done to compare the differences of MAD measurements for each method. The comparative analysis were made between these two groups using Mann-Whitney test for $\mathrm{MAD}_{\mathrm{L}}$ and $\mathrm{MAD}_{\mathrm{S}}$ values. The variance in Mean $\pm S D$ values of $M_{A} D_{L}$ and $M A D_{S}$ between Group1 and Group2 are presented in Table 3.

Accordingly, the statistical results of the comparison between Group1 and Group2 using the 'Mann-Whitney' test showed no significant differences between displacement measurements for $\operatorname{MAD}_{\mathrm{L}}(P=0.55)$ and $\mathrm{MAD}_{\mathrm{S}}(P=0.61)$. In Fig. 7 , the comparative analysis results of the $\mathrm{MAD}_{\mathrm{S}}$ and $\mathrm{MAD}_{\mathrm{L}}$ measurements are shown. Based on these experiments, both qualitative and quantitative assessments were found the results of Group1 and Group2 to be comparable. Therefore, the proposed method for displacement measurements is justified based on the finding of resolutions calculations and can be considered as an accurate method. 


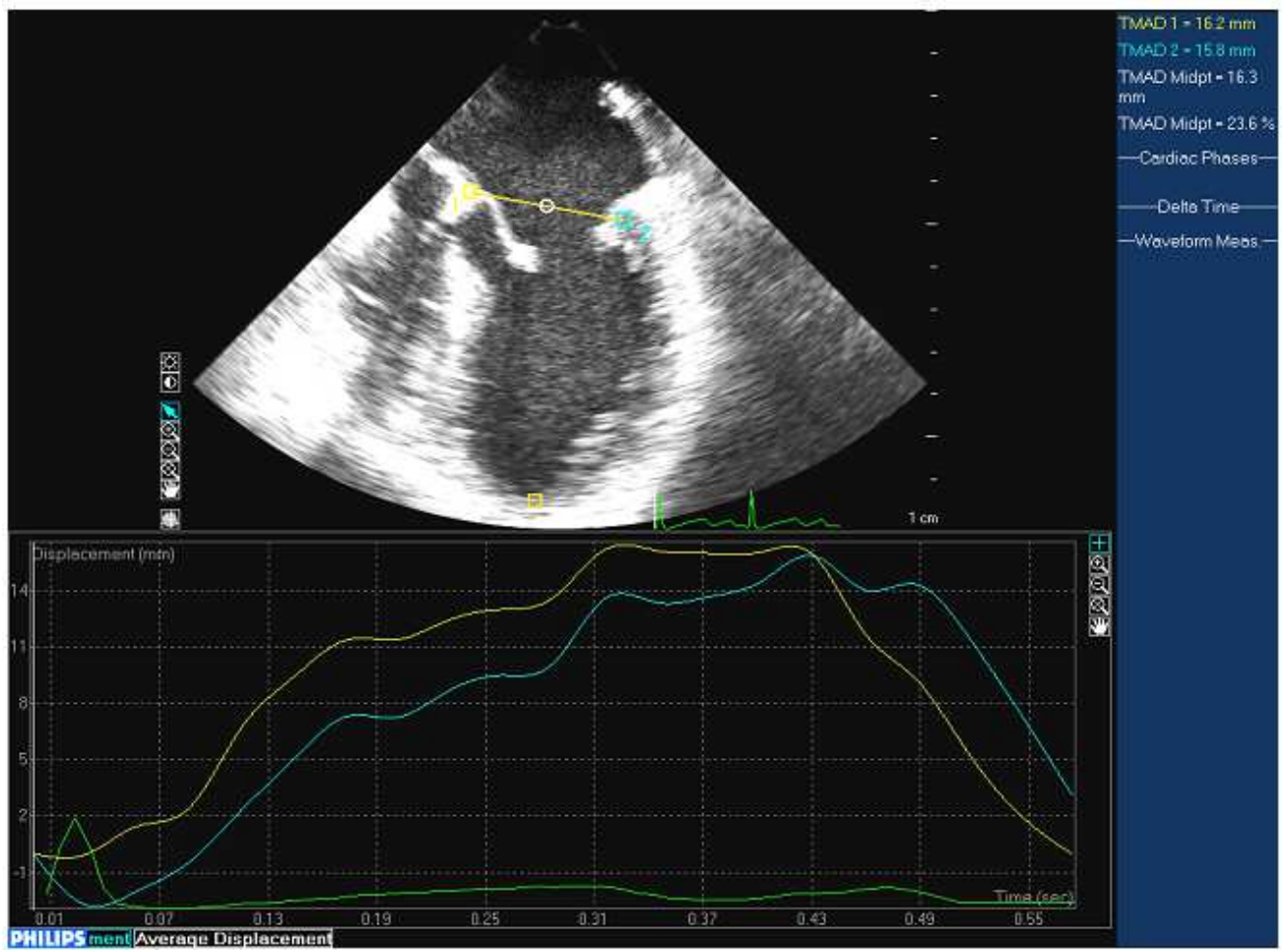

Fig. 5. The results of TMAD measurement using 'TMQ Advanced' technique by QLAB software

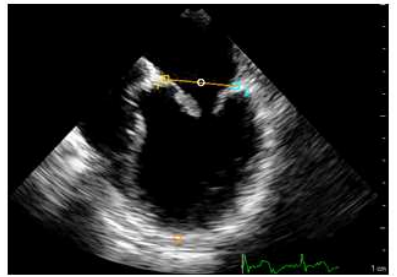

(a)

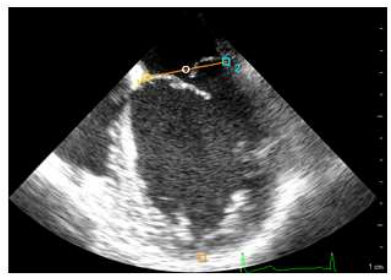

(b)

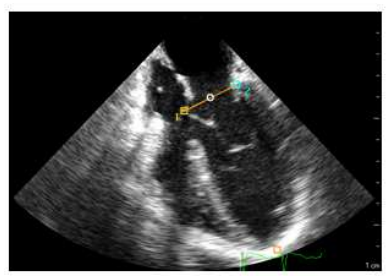

(c)

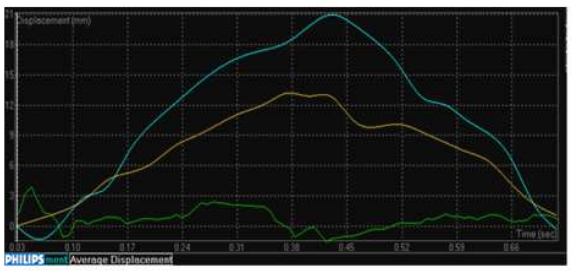

(d)

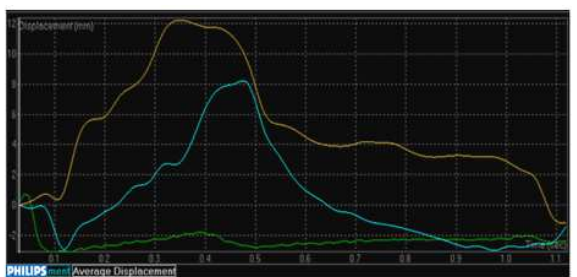

(e)

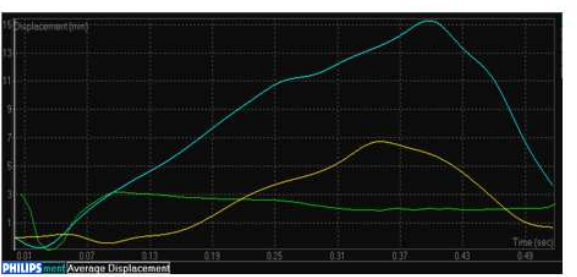

(f)

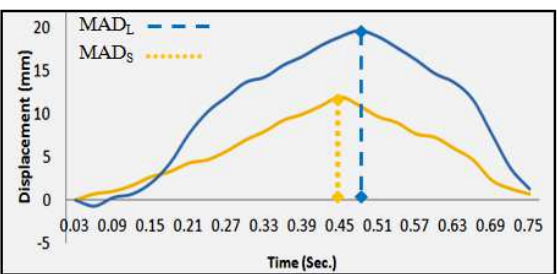

(g)

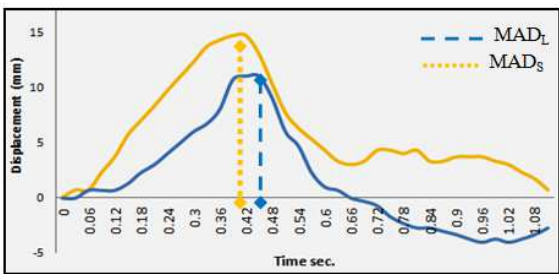

(h)

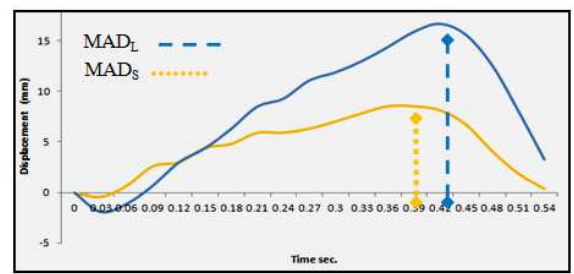

(i)

Fig. 6. Several examples of the mitral annular displacement measurements with Time/curve in 4-chamber view of 2Dechocardiography images (a-c) using 'TMQ Advanced' technique by QLAB software (d-f) and the proposed method of MAD measurements (g-i) 


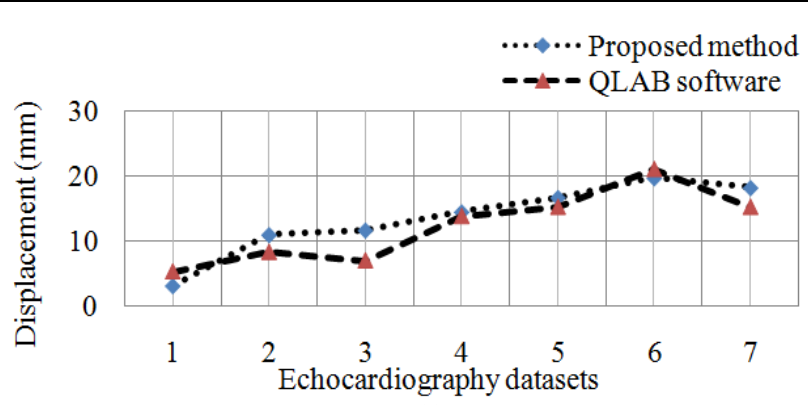

(a)

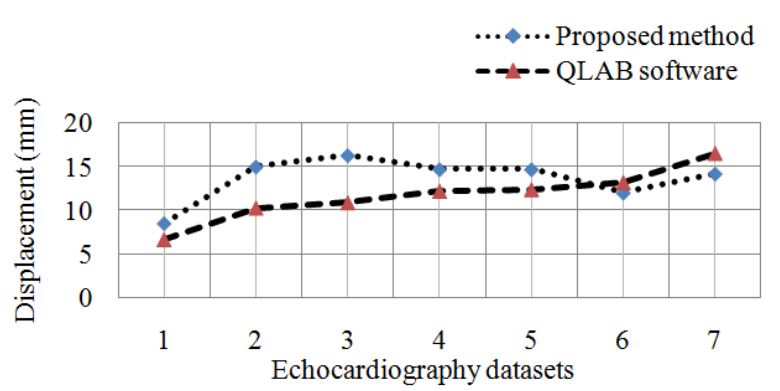

(b)

Fig. 7. The comparative analysis results of MADL (a) and MADS (b) measurements to all datasets using the proposed method and QLAB software

Table 1. The qualitative assessment for MAD measurements by visual observation of two experts with their check mark in black and red colour

\begin{tabular}{|c|c|c|c|}
\hline \multirow{2}{*}{$\begin{array}{l}\text { 2DE images } \\
\mathrm{N}=178\end{array}$} & \multicolumn{3}{|c|}{ Evaluation scores } \\
\hline & Low & Acceptable & High \\
\hline $\mathrm{Pt} \# 1$ & & & $\sqrt{ } \sqrt{ }$ \\
\hline $\mathrm{Pt} \# 2$ & & $\sqrt{ }$ & $\sqrt{ }$ \\
\hline $\mathrm{Pt} \# 3$ & & $\sqrt{ } \sqrt{ }$ & \\
\hline $\mathrm{Pt} \# 4$ & & & $\sqrt{ } \sqrt{ }$ \\
\hline $\mathrm{Pt} \# 5$ & & & $\sqrt{ } \sqrt{ }$ \\
\hline $\mathrm{Pt} \# 6$ & & & $\sqrt{ } \sqrt{ }$ \\
\hline $\mathrm{Pt} \# 7$ & & & $\sqrt{ } \sqrt{ }$ \\
\hline
\end{tabular}

Table 2. The $\mathrm{MAD}_{\mathrm{L}}$ and $\mathrm{MAD}_{\mathrm{s}}$ measurement values in millimetres (mm)

\begin{tabular}{|c|c|c|c|c|}
\hline \multirow[b]{2}{*}{ 2DE datasets } & \multicolumn{2}{|l|}{ Group1 } & \multicolumn{2}{|c|}{ Group2 } \\
\hline & $\mathrm{MAD}_{\mathrm{L}}$ & $\mathrm{MAD}_{\mathrm{S}}$ & $\mathrm{MAD}_{\mathrm{L}}$ & $\mathrm{MAD}_{\mathrm{S}}$ \\
\hline$\overline{\mathrm{Pt} \# 1}$ & 14.5 & 14.2 & 15.8 & 16.5 \\
\hline $\mathrm{Pt} \# 2$ & 3.8 & 8.4 & 5.3 & 10.9 \\
\hline $\mathrm{Pt} \# 3$ & 11.0 & 14.7 & 8.0 & 12.2 \\
\hline $\mathrm{Pt} \# 4$ & 16.7 & 8.5 & 15.2 & 6.7 \\
\hline $\mathrm{Pt} \# 5$ & 11.7 & 17.3 & 7.3 & 12.4 \\
\hline $\mathrm{Pt} \# 6$ & 19.7 & 12.0 & 20.6 & 13.2 \\
\hline $\mathrm{Pt} \# 7$ & 19.5 & 15.0 & 13.7 & 10.3 \\
\hline
\end{tabular}

Table 3. The quantitative assessment for MAD measurements by statistical measurements of Mean $\pm \mathrm{SD}$

\begin{tabular}{lll}
\hline $\mathrm{N}=7$ & Group1 Mean $\pm \mathrm{SD}$ & Group2 Mean $\pm \mathrm{SD}$ \\
\hline $\mathrm{MAD}_{\mathrm{L}}$ & $13.78 \pm 5.81$ & $12.82 \pm 5.63$ \\
$\mathrm{MAD}_{\mathrm{S}}$ & $12.58 \pm 3.17$ & $11.73 \pm 2.91$ \\
\hline
\end{tabular}

\section{Conclusion}

In this study, the main aim is to measure the mitral annular displacement MAD based on Septal and Lateral motion tracking through a complete cardiac cycle in 2Dechocardiography images. In clinical practice, MAD measurement is a reproducible method of determining the LV global systolic function and could be an easily detectable index for wall motion abnormalities. A computational method of MAD measurements was proposed based on the findings to estimate the image resolution. The comparative analysis of displacement measurements have been done qualitatively and quantitatively between the proposed method of MAD and TMAD measurements by QLAB software. In the context of qualitative assessment, the results clearly demonstrated that the MAD measurements has high acceptability of $85 \%$ according to the opinion of two experts by visual observation. Furthermore, the quantitative analysis shows that the MAD measurements method is comparable with TMAD measurements by QLAB and there is no significant differences in displacement measurements.

\section{Acknowledgement}

The authors would like to thank both of Dr. IKA FAIZURA (Clinical cardiologists in National Heart Institute IJN, Malaysia) and Mrs. NORLAILA DANURI (Cardiac Technician, Cardiology Unit, PPUKM hospital) for their useful assistances in evaluate the results of our proposed method by visual observation. Also, special thanks to Mr. MOHD FAIZAL (Seiner Manager, Clinical Research Dept. in IJN) for his assistance and advice in statistical analysis.

\section{Author's Contributions}

Faten A. Dawood: Designed the research plan and organized the study. Coordinated the overall framework and participated in all experiments, data-analysis as well as contributed to the writing of the manuscript.

Rahmita W. Rahmat: Designed the research plan and organized the study. Participated in all experiments, coordinated the overall framework and contributed to the writing of the manuscript.

Suhaini B. Kadiman: Designed the research plan and organized the study. Participated in all clinical experiments, coordinated the data-acquisition and analysis. 
Lili N. Abdullah: Participated in the research plan and organized the study. Reviewing the research and participated in the results analysis.

Mohd D. Zamrin: Participated in the research plan. Coordinated in the comparative study of experiments and the results analysis.

\section{Ethics}

This article is original and contains unpublished material. The corresponding author confirms that all of the other authors have read and approved the manuscript and no ethical issues involved.

\section{References}

Ahanathapillai, V. and J.J. Soraghan, 2010. Myocardial Ischemia Detection Algorithm (MIDA): Automated echocardiography sequence analysis for diagnosis of heart muscle damage. Proceedings of the Computing in Cardiology, Sept. 26-29, IEEE Xplore Press, Belfast, pp: 405-408.

Ahmed, H.S. and M.J. Nordin, 2011. Improving diagnostic viewing of medical images using enhancement algorithms. J. Comput. Sci., 7: 1831-1838.

DOI: $10.3844 /$ jcssp.2011.1831.1838

Al-Surmi, A., O. Rahmat, R. Wirza, M.Z. Dimon and R. Mahmod et al., 2013. Three dimensional reconstruction of human heart surface from single image-view under different illumination conditions. Am. J. Applied Sci., 10: 669-680. DOI: 10.3844/ajassp.2013.669.680

Black, D.E., J. Bryant, C. Peebles, K.M. Godfrey and M. Hanson et al., 2013. Tissue motion annular displacement of the mitral valve using twodimensional speckle tracking echocardiography predicts the left ventricular ejection fraction in normal children. Cardiol. Young, 24: 640-648. PMID: 23803408

Buss, S.J., D. Mereles, M. Emami, G. Korosoglou and J.H. Riffel et al., 2012. Rapid assessment of longitudinal systolic left ventricular function using speckle tracking of the mitral annulus. Clinical Res. Cardiol., 101: 273-280. PMID: 22139127

Dawood, F.A., R.W. Rahmat, S.B. Kadiman, L.N. Abdullah and M.D. Zamrin, 2012. Effect comparison of speckle noise reduction filters on 2DEchocardigraphic images. World Acad. Sci. Eng. Technol., 69: 425-430.

Dawood, F.A.A., R.W. Rahmat, M.Z. Dimon, L. Nurliyana and S.B. Kadiman, 2011. Automatic boundary detection of wall motion in twodimensional echocardiography images. J. Comput. Sci., 7: 1261-1266.

DOI: $10.3844 /$ jcssp.2011.1261.1266
DeCara, J.M., E. Toledo, I.S. Salgo, G. Lammertin and L. Weinert et al., 2005. Evaluation of left ventricular systolic function using automated angle-independent motion tracking of mitral annular displacement. J. Am. Society Echocardiogr., 18: 1266-1269. PMID: 16376753

dos Reis, M.D.C., A.F. da Rocha, D.F. Vasconcelos, B.L. Espinoza and F.A.d.O. Nascimento et al., 2008. Semi-automatic detection of the left ventricular border. Proceedings of the 30th Annual International Conference of the IEEE Engineering in Medicine and Biology Society, Aug. 20-25, IEEE Xplore Press, Vancouver, BC, pp: 218-221. DOI: 10.1109/IEMBS.2008.4649129

Emilsson, K., M. Alam and B. Wandt, 2000. The relation between mitral annulus motion and ejection fraction: A nonlinear function. J. Am. Society Echocardiogr., 13: 896-901. PMID: 11029713

Eto, M., K. Toba, M. Akishita, K. Kozaki and To. Watanabe et al., 2005. Impact of blood pressure variability on cardiovascular events in elderly patients with hypertension. Hypertension Res., 28: 1-7. DOI: 10.1291/hypres.28.1

Hedberg, P., T. Jonason, I. Lönnberg, G. Nilsson and K. Pehrsson et al., 2006. Mitral annulus motion as a predictor of mortality in a community-based sample of 75-year-old men and women. J. Am. Society Echocardiogr., 19: 88-94. PMID: 16423675

Hoque, M.R. and M. Rashed-al-Mahfuz, 2011. A new approach in spatial filtering to reduce speckle noise. Int. J. Soft Comput. Eng., 1: 29-32.

Ito, K., M. Noma, M. Mohri, K. Abe and U. Yamamoto et al., 2007. Mitral annulus displacement measured by tissue-tracking method with Doppler-tissue images is a useful marker of the severity of heart failure. J. Cardiol., 50: 159-166. PMID: 17941191

Jagatheeswari, P., S. Suresh Kumar and M. Rajaram, 2009. Contrast enhancement for medical images based on histogram equalization followed by median filter.

Nandagopalan, S., B. Adiga, C. Dhanalakshmi and N. Deepak, 2010. Automatic segmentation and ventricular border detection of 2D echocardiographic images combining k-means clustering and active contour model. Proceedings of the Second International Conference on Computer and Network Technology, Apr. 23-25, IEEE Xplore Press, Bangkok, pp: 447-451. DOI: 10.1109/ICCNT.2010.110

Narayanan, A., J.C. Hill and G.P. Aurigemma, 2007. Tissue mitral annular displacement-a novel descriptor of global left ventricular function. Radcliffe Cardiology. 
Pan, C., R. Hoffmann, H. Kühl, E. Severin and A. Franke et al., 2001. Tissue tracking allows rapid and accurate visual evaluation of left ventricular function. Eur. J. Echocardiogr., 2: 197-202. PMID: 11882453

Rahmat, R.W., F.A. Dawood, S.B. Kadiman, L.N. Abdullah and M.D. Zamrin, 2012. border detection of ventricle wall motion in echocardiographic images: A survey. Proceedings of the International Conference on Advanced Computer Science Applications and Technologies, Nov. 26-28, IEEE Xplore Press, Kuala Lumpur, pp: 486-490. DOI: 10.1109/ACSAT.2012.72

Salvador, A., Y. Maingourd, S. Fu and J.F. Lerallut, 2003. Optimization of an edge detection algorithm for echocardiographic images. Proceedings of the 25th Annual International Conference of the IEEE Engineering in Medicine and Biology Society, Sept. 17-21, IEEE Xplore Press, pp: 1188-1191.

DOI: $10.1109 /$ IEMBS.2003.1279462
Storaa, C., P. Cain, B. Olstad, B. Lind and L.Å. Brodin, 2004. Tissue motion imaging of the left ventriclequantification of myocardial strain, velocity, acceleration and displacement in a single image. Eur. J. Echocardiogr., 5: 375-385. PMID: 15341873

Sun, J.P., A.P.W. Lee, C. Wu, Y.Y. Lam and M.J. Hung et al., 2013. Quantification of left ventricular regional myocardial function using two-dimensional speckle tracking echocardiography in healthy volunteers-a multi-center study. Int. J. Cardiol., 167: 495-501. PMID: 22365315

Zahid, W., J. Johnson, C. Westholm, C.H. Eek and K.H. Haugaa et al., 2013. Mitral annular displacement by doppler tissue imaging may identify coronary occlusion and predict mortality in patients with non-ST-elevation myocardial infarction. J. Am. Society Echocardiogr., 26: 875-884. PMID: 23791116 